\title{
Photoacoustic optical frequency comb spectroscopy of radioactive methane in the mid-infrared region
}

\section{Karhu, Juho}

\section{IEEE}

2019

Karhu , J , Tomberg , T , Vieira , F S , Genoud , G , Hänninen , V , Vainio , M , Metsälä , M , Hieta , T , Bell , S \& Halonen , L 2019 , Photoacoustic optical frequency comb spectroscopy of radioactive methane in the mid-infrared region . in 2019 Conference on Lasers and Electro-Optics Europe \& European Quantum Electronics Conference (CLEO/Europe-EQEC) . IEEE , The 2019 Conference on Lasers \& Electro-Optics / Europe and the European Quantum Electronics Conference (CLEO®/Europe-EQEC) , München , Germany , 23/06/2019 . https://doi.org/10.1109/CLEOE-EQEC.2019.8872587

http://hdl.handle.net/10138/324431

https://doi.org/10.1109/CLEOE-EQEC.2019.8872587

acceptedVersion

Downloaded from Helda, University of Helsinki institutional repository.

This is an electronic reprint of the original article.

This reprint may differ from the original in pagination and typographic detail.

Please cite the original version. 


\title{
Photoacoustic Optical Frequency Comb Spectroscopy of Radioactive Methane in the Mid-Infrared Region
}

\author{
Juho Karhu ${ }^{1}$, Teemu Tomberg ${ }^{1}$, Francisco Senna Vieira ${ }^{1,2}$, Guillaume Genoud ${ }^{3}$, Vesa Hänninen ${ }^{1}$, Markku \\ Vainio $^{1,4}$, Markus Metsälä ${ }^{1}$, Tuomas Hieta ${ }^{5}$, Steven Bell ${ }^{6}$, Lauri Halonen ${ }^{1}$ \\ 1. Department of Chemistry, University of Helsinki, Helsinki, Finland \\ 2. Currently with the Department of Physics, Umeå University, Umeå, Sweden \\ 3. Centre for Metrology MIKES, VTT Technical Research Centre of Finland Ltd., Finland \\ 4. Photonics Laboratory, Physics Unit, Tampere University, Tampere, Finland \\ 5. Gasera Ltd., Turku, Finland \\ 6. National Physical Laboratory, Teddington, UK
}

The standard method for sensitive measurements of long-lived radioactive isotopes, such as ${ }^{14} \mathrm{C}$, is accelerator mass spectrometry (AMS). The AMS facilities are indispensable for applications like radiocarbon dating, but too expensive and massive for in-situ measurements. Laser spectroscopy, on the other hand, is a promising solution for isotope-selective detection of gas-phase compounds in applications that require low-cost instrumentation and on-site measurement capability. The recent work on laser spectroscopy of ${ }^{14} \mathrm{CO}_{2}$ has led to impressive detection limits below the natural ${ }^{14} \mathrm{C}$ abundance [1,2], which encourages us to develop similar techniques also for other radiocarbon compounds. Here, we report the first spectroscopic study of radioactive methane, ${ }^{14} \mathrm{CH}_{4}$. Our work is motivated by a number of important applications, such as determining the biofraction of methane fuel mixtures and in-situ monitoring of radioactive gas emissions at decommissioning sites of light water nuclear reactors.

An essential prerequisite for the development of optical detection of ${ }^{14} \mathrm{CH}_{4}$ is to compile a spectroscopic database with relevant absorption line parameters. Here, we report the first measurement of ${ }^{14} \mathrm{CH}_{4}$ absorption spectrum. We focus on the fundamental antisymmetric $\mathrm{CH}$-stretching vibrational band $\left(\mathrm{v}_{3}\right)$ of methane in the $3 \mu \mathrm{m}$ wavelength region. Our results make it possible to locate the absorption lines that are most suitable for ${ }^{14} \mathrm{CH}_{4}$ detection and quantification while minimizing the spectral interference due to other molecular compounds.

In the measurements of ${ }^{14} \mathrm{CH}_{4}$ absorption spectrum we have used a recently introduced technology, which combines a broadband optical frequency comb (OFC) light source with Fourier-transform interferometry and cantilever-enhanced photoacoustic spectroscopy (CEPAS) [3,4]. The CEPAS method provides an excellent spectroscopic detection sensitivity with a small sample volume of only a few millilitres, which is particularly advantageous when the sample amount is limited, such as in the case of radioactive species. The mid-infrared OFC light source is a high-power femtosecond optical parametric oscillator, which allows us to perform sensitive (sub $100 \mathrm{ppb}$ ) broadband measurements of the entire $\mathrm{v}_{3}$ bands of ${ }^{12} \mathrm{CH}_{4}$ and ${ }^{14} \mathrm{CH}_{4}$. Not only have we demonstrated the first optical measurements of ${ }^{14} \mathrm{CH}_{4}$, but also an order of magnitude improvement in trace gas detection sensitivity compared to the best previously reported broadband photoacoustic measurements.

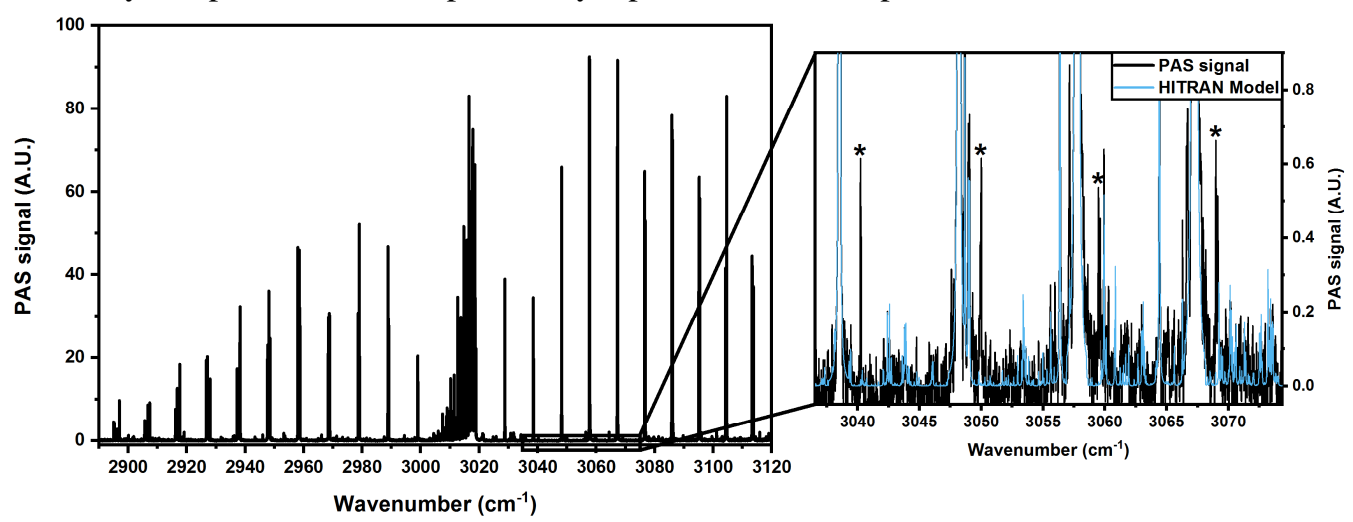

Fig. 1. Photoacoustic spectrum of the ${ }^{14} \mathrm{CH}_{4}$ sample (about $100 \mathrm{ppm}{ }^{12} \mathrm{CH}_{4}$ and $1 \mathrm{ppm}{ }^{14} \mathrm{CH}_{4}$, with residual water content). The inset shows some identified ${ }^{14} \mathrm{CH}_{4}$ lines $\left(^{*}\right)$ which are absent in the HITRAN model of ${ }^{12} \mathrm{CH}_{4}$ and water. The observed line positions are in agreement with our theoretical calculation of the isotope shift.

\section{References}

[1] I. Galli, S. Bartalini, R. Ballerini, M. Barucci, P. Cancio, M. De Pas, G. Giusfredi, D. Mazzotti, N. Akikusa, and P. De Natale, "Spectroscopic detection of radiocarbon dioxide at parts-per-quadrillion sensitivity," Optica. 3, 385 (2016).

[2] G. Genoud, M. Vainio, H. Phillips, J. Dean, and M. Merimaa, "Radiocarbon dioxide detection based on cavity ring-down spectroscopy and a quantum cascade laser," Opt. Lett.40, 1342 (2015).

[3] T. Mikkonen, C. Amiot, A. Aalto, K. Patokoski, G. Genty, and J. Toivonen, "Broadband cantilever-enhanced photoacoustic spectroscopy in the mid-IR using a supercontinuum," Opt. Lett. 43, 5094 (2018).

[4] I. Sadiek, T. Mikkonen, M. Vainio, J. Toivonen, and A. Foltynowicz, "Optical frequency comb photoacoustic spectroscopy," Phys

Chem. Chem. Phys. 20, 27849 (2018).

(C) 2021 IEEE. Personal use of this material is permitted. Permission from IEEE must be obtained for all other uses, in any current or future media, including reprinting/republishing this material for advertising or promotional purposes, creating new collective works, for resale or redistribution to servers or lists, or reuse of any copyrighted component of this work in other works. 